\title{
IMAGING SPECTROPHOTOMETRY OF THE NARROW-LINE REGION OF NGC 1068
}

\author{
GERALD CECIL \\ Institute for Advanced Study \\ Princeton \\ NJ 08540 \\ JOSS BLAND \\ Institute for Astronomy \\ Honolulu \\ HI 96822
}

ABSTRACT. The NLR of the Sy 2 galaxy NGC 1068 has been studied with the Hawaii Imaging Fabry-Perot Interferometer on the U. Hawaii $2.2 \mathrm{~m}$ telescope. Kinematics of [N II] $\lambda \lambda 6548,6583$ and $\mathrm{H} \alpha$ were mapped across the inner $1^{\prime}$-diameter by obtaining monochromatic images ( $\left.65 \mathrm{~km} \mathrm{~s}^{-1} \mathrm{FWHM}\right)$ with a low-noise (5e $\left.\mathrm{e}^{-} \mathrm{RMS}\right) \mathrm{CCD}$ at sub-arcsecond spatial and $\sim 90 \mathrm{~km} \mathrm{~s}^{-1}$ effective velocity resolution. Our complete coverage of this nearby ( $14 \mathrm{Mpc} ; 1^{\prime \prime}=75 \mathrm{pc}$ ), spatially extended NLR allows unprecedented study of the interaction of a collimated radio "jet" (Wilson \& Ulvestad 1983) with the warm ionized component of the ambient interstellar medium.

\section{Results}

Our line profiles were synthesized from image stacks at $0 . " 5$ spatial increments, are in excellent agreement with those obtained by other researchers (Walker 1968; Meaburn \& Pedlar 1986; Baldwin et al. 1987), and are being analyzed for kinematic and spectrophotometric content. Figure 1 shows representative profiles, our working kinematic decomposition into Gaussian subsystems, and the $\lambda-6 \mathrm{~cm}$ continuum map. The intensity of high-velocity line emission varies by nearly three orders of magnitude over the region shown, so a CCD detector is essential. In addition, we use an elaton with a freespectral range of $4000 \mathrm{~km} \mathrm{~s}^{-1}$ to avoid inter-order confusion.

By summing our monochromatic images after subtracting the fitted continuum level at each point, we generate an image equivalent to that obtained with traditional narrow-band interference filters. The NLR is aligned along the jet axis, but the correspondence is not tight. However, when the spectra are decomposed into the Gaussian components shown in Figure 1, specific kinematic subsystems noted by other observers at a few points in their long-slit datasets can be tracked across the entire NLR, and a striking resemblance to certain radio structures is now evident. Sharply defined, bright features exhibit sharp kinematic boundaries, and thus resemble not static, photoionized nebulae, but rather the termini of hydrodynamic flows.

High-velocity gas is distributed a): to the NE, in the wings of the emission lines (first mapped by Walker 1968); and, b) everywhere else, as a broad component first isolated by Pelat and Alloin (1980). The centroids of the former components are typically displaced approximately $500 \mathrm{~km} \mathrm{~s}^{-1}$ from systemic velocity, and exhibit (equal) half-widths of the same order of magnitude. The broad component has a FWHM of $\sim 1200 \mathrm{~km} \mathrm{~s}^{-1}$, and extends throughout much of the inner 4 " radius. Overall, the high345 
velocity $[\mathrm{N} \mathrm{II]} \mathrm{is} \mathrm{aligned} \mathrm{along} \mathrm{the} \mathrm{jet,} \mathrm{and} \mathrm{rapidly} \mathrm{diminishes} \mathrm{in} \mathrm{intensity} \mathrm{when} \mathrm{the} \mathrm{jet} \mathrm{decollimates} \mathrm{at}$ hotspots NE and SW of the nucleus. The line profiles in this region are double-peaked. Gas velocities are at least $500 \mathrm{~km} \mathrm{~s}^{-1}$ with respect to systemic, and higher once projection effects are accounted for. Near systemic velocity, the narrow kinematic "flare" is found in the NE quadrant. Its brightest emission coincides with the NE radio lobe, attaining peak brightness along the NW (leading) edge with respect to galactic rotation. This brightening is also apparent in our $\mathrm{H} \alpha$ datacube, and so is a mass enhancement. $\mathrm{SW}$ of the nucleus an optical hot-spot peaks in a region of deficient radio flux. This emission component is broadened to several hundred $\mathrm{km} \mathrm{s}^{-1}$. Further down the jet, however, optical emission peaks in a radio hotspot, with no evidence of line broadening. Much of the nuclear flux is contained in the spatially extended, nuclear component which has FWHM of $-600 \mathrm{~km} \mathrm{~s}^{-1}$. It dominates the other components within $1^{\prime \prime}$ of the nucleus, rendering the decomposition ambiguous.

\section{$\Delta \delta(")$}

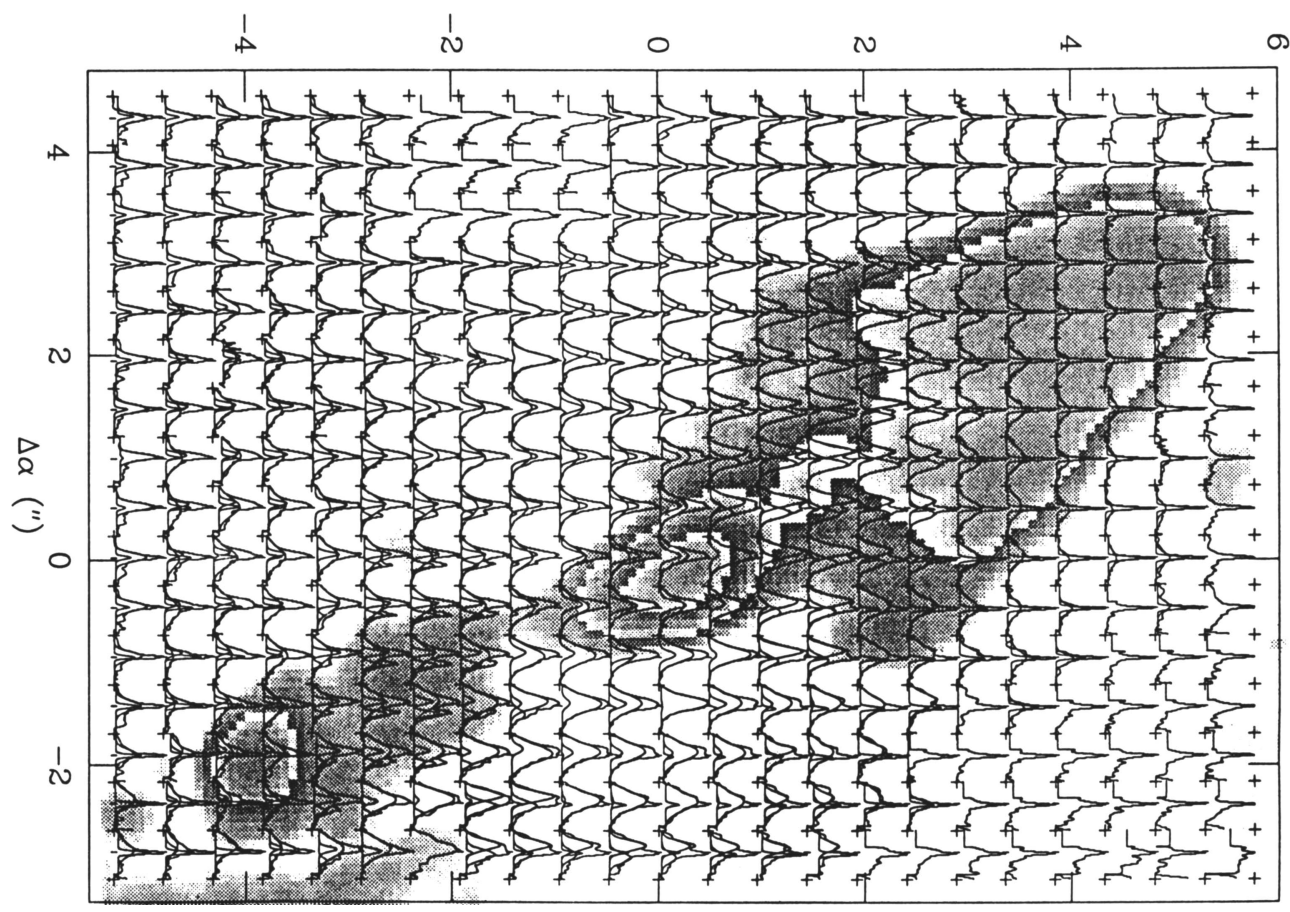

Figure 1. [N II] line profiles over the NLR of NGC 1068. Each spectrum is intensity normalized in a box $0 . " 5$ on a side and $3200 \mathrm{~km} \mathrm{~s}^{-1}$ in extent. Gaussian components are superposed, together with the $\lambda-6 \mathrm{~cm}$ continuum. South to left, east at top.

\section{References}

Baldwin, J.A., Wilson, A.S., and Whittle, M. 1987, Ap. J., 319, 84.

Meaburn, J., and Pedlar, A. 1986, Astr. Ap., 159, 336.

Pelat, D., and Alloin, D. 1980, Astr. Ap., 81, 172.

Walker, M.F. 1968, Ap. J., 151, 71.

Wilson, A.S., and Ulvestad, J.E. 1983, Ap. J., 275, 8. 